\title{
The Initial Contraction of Skinned Muscle Fibers on Photorelease of ATP in the Presence of ADP
}

\author{
Keisuke HoRIUTI*, Koichi KaGAwa, and Kazuhiro Yamada \\ Department of Physiology, Oita Medical University, \\ Oita, 879-55 Japan
}

\begin{abstract}
We photoreleased ATP in skinned muscle fibers from rat psoas, and examined the effects of ADP on the ATP-induced isometric transient. 2) On release of ATP, the muscle stiffness initially falls at any $\left[\mathrm{Ca}^{2+}\right]$. ADP slowed this stiffness decrease, suggesting competition between ADP and ATP. Even in the presence of ADP, the rate of stiffness decrease was proportional to the amount of released ATP, but was not affected by $\mathrm{Ca}^{2+}, \mathrm{P}_{\mathrm{i}}$, or the fiber tension. 3) In the absence of ADP, the $\mathrm{Ca}^{2+}$ sensitive contraction occurs only after the initial phase. However, in the presence of ADP, the fiber developed tension during the initial phase before the late contraction. The initial contraction was insensitive to $\mathrm{Ca}^{2+}$, resistant to $\mathrm{P}_{\mathrm{i}}$ and $\mathrm{BDM}$, larger on releasing more ATP, but not markedly influenced by the fiber tension. 4) Our results seem to suggest that, in the physiological reaction cycle, the cross-bridges develop significant force immediately after the binding of ATP, before releasing $\mathbf{P}_{\mathrm{i}}$.
\end{abstract}

Key words : $\quad$ skeletal muscle, cross-bridge, caged ATP, photolysis, $\mathrm{Ca}^{2+}$.

The cross-bridge, i.e. the complex of the myosin head and actin in muscle fiber, produces contractile force by hydrolyzing ATP into ADP plus $P_{i}$. Physiological and biochemical studies so far generally indicate the following cycle of cross-bridge reaction (see introduction of [1] for refs.):

$$
\begin{aligned}
& \underset{(\mathrm{ADP} \cdot \mathrm{R})}{\mathrm{AM} \cdot \mathrm{ADP}} \stackrel{-\mathrm{ADP}}{\rightleftarrows} \mathrm{AM} \stackrel{+\mathrm{ATP}}{\longrightarrow}\left\{\begin{array}{ll}
\mathrm{AM} \cdot \mathrm{ATP} & \mathrm{AM} \cdot \mathrm{ADP} \cdot \mathrm{P}_{\mathrm{i}} \\
\mathrm{A} / \mathrm{M} \cdot \mathrm{ATP} & \mathrm{A} / \mathrm{M} \cdot \mathrm{ADP} \cdot \mathrm{P}_{\mathrm{i}}
\end{array}\right\} \\
& \stackrel{-\mathbf{P}_{\mathrm{i}}}{\rightleftarrows} \mathrm{AM}^{*} \cdot \mathrm{ADP} \longrightarrow \cdots \\
& \text { (F) }
\end{aligned}
$$

In the absence of ATP, myosin heads (M) are tightly bound to actin (A) to form the rigor cross-bridges $(\mathrm{R})$. On binding ATP, the cross-bridges enter the weak-

Received on September 1, 1994; Accepted on October 14, 1994

* To whom correspondence should be addressed. 
binding state (W), where the interaction between myosin and actin is rapid but so weak that they are substantially dissociated from each other. ATP is quickly hydrolyzed into ADP $+\mathrm{P}_{i}$ on myosin in this state. The strong-binding, forcegenerating state $(F)$ lies next to state $W$, and $P_{i}$ release is closely related with the W-to-F transition (see [2,3] for details of $P_{i}$ release). The regulatory action of $\mathrm{Ca}^{2+}$ is assumed to be mainly from the $\mathrm{Ca}^{2+}$-sensitivity of this weak-to-strong transition [4]. After force generation, the cross-bridges are slowly isomerized to rigor cross-bridges that still hold ADP (ADP $\cdot \mathbf{R})$. The ADP $\cdot \mathbf{R}$ cross-bridges soon release ADP to return to the nucleotide-free rigor state $(R)$. The contractile force induced at the previous W-to-F transition is kept until this stage, and is lost at the following R-to-W transition in the next cycle. In this scheme, when one adds ADP in the system, the ADP acts as a competitive inhibitor for the binding of ATP.

By photolysis of caged ATP in skinned muscle fibers, one can examine the ATP-induced mechanical response of the cross-bridges [5,6]. Much evidence for the above scheme was obtained in such studies with caged ATP (see [1]). Dantzig and colleagues [1] studied the effects of ADP on the ATP-induced reaction in muscle fibers. Recently, we have conducted a similar study of ADP, but at a low temperature and at various $\left[\mathrm{Ca}^{2+}\right]$. We have made several new findings and obtained some conclusions which are different from those by the previous authors [1]. We suggest that the replacement of ADP with ATP (ADP $\cdot R \stackrel{-A D P}{\longrightarrow} R \stackrel{+ \text { ATP }}{\longrightarrow}$ W) is a physiological force-generating step, which is insensitive to $\mathrm{Ca}^{2+}$ and $\mathbf{P}_{\mathrm{i}}$. An abstract has appeared elsewhere [7].

\section{METHODS}

The materials, setup, and procedures were the same as previously described [8]. The skinned fibers were prepared from rat psoas muscle at $20^{\circ} \mathrm{C}$, stored in the relaxing solution at $4^{\circ} \mathrm{C}$, and used within $36 \mathrm{~h}$. A segment of a muscle fiber was mounted on the setup for experiment. At each trial in the experiment, the fiber segment was first put into the rigor state by washing it with an ATP-free (rigor) solution, and then transferred to a trough filled with a solution containing caged ATP. After equilibration for 30-60s, a UV flash was applied to the fiber to release ATP from caged ATP, and the mechanical transient on photolysis was recorded for later analysis. The photolysis trials were carried out at 7-9 ${ }^{\circ} \mathrm{C}, \mathrm{pH} 7.0$, and an ionic strength of $0.20 \mathrm{M}$. $\mathrm{Ca}^{2+}$ was buffered with $10 \mathrm{~mm}$ EGTA. ADP and other agents were added in the photolysis solution when required. The muscle fibers were kept isometric during photolysis transient, but in some cases they were stretched by about $1 \%$ several seconds before photolysis, to increase the prephotolysis rigor tension. The muscle stiffness was monitored throughout the experiments by applying a length oscillation $(500 \mathrm{~Hz}, 0.1-0.15 \%$ [5,9]). The "maximum active tension" in RESULTS refers to the steady-state tension obtained by ATP release in the presence of $10 \mathrm{mM} \mathrm{Ca} \cdot$ EGTA $(\mathrm{pCa} \approx 4.5)$ without ADP and $P_{i}$.

The amounts of ATP photoreleased from caged ATP was examined by 
chromatographic analyses. When the flash energy of the xenon lamp for photolysis was the maximum $(200 \mathrm{~J})$, the photolysis efficiency was $17-25 \%$ in this study. The efficiency was reduced, when required, by decreasing the flash energy to a half or a quarter. The rate of ATP release was estimated to be about $40 \mathrm{~s}^{-1}$ at our experimental temperature, $\approx 8^{\circ} \mathrm{C}$ : it has been reported [5] that the rate is $118 \mathrm{~s}^{-1}$ at $21^{\circ} \mathrm{C}$ with $Q_{10}=2.3$. Caged ATP used in this study was the NPE-caged ATP $\left(\mathbf{P}^{3}\right.$ -

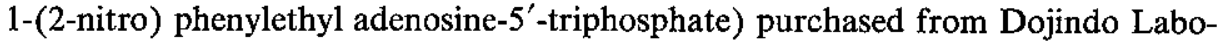
ratory (Japan), and contaminant ATP in this batch was reported to be $0.026 \%$ of caged ATP. K·ADP (type $[\mathrm{II}]$ ) and $\mathrm{AP}_{5} \mathrm{~A}\left(\mathbf{P}^{1}, \mathbf{P}^{5}\right.$-di(adenosine-5')-pentaphosphate) were from Boehringer-Mannheim-Yamanouchi (Japan).

In RESULTS, the figures with \pm signs after the mean values represent SEMs, and the $n$ values in parentheses indicate the numbers of the examined fibers, unless otherwise stated.

\section{RESULTS}

$A T P$-induced transient in stiffness and effects of $A D P$

Upper panel of Fig. 1 shows the stiffness transients induced by photorelease of $0.84 \mathrm{~mm}$ ATP at three different $\left[\mathrm{Ca}^{2+}\right]$ : a supramaximal $(\mathrm{pCa} \approx 4.5)$, an intermediate ( $\mathrm{pCa} 6.6)$, and an extremely low $\left[\mathrm{Ca}^{2+}\right](\mathrm{pCa}>8)$. We added $12 \mathrm{mM} \mathbf{P}_{\mathrm{i}}$ in the low $\left[\mathrm{Ca}^{2+}\right]$ solution to suppress the contractile activity [10]. On release of ATP, stiffness initially decreased, the time course of which was almost independent of $\left[\mathrm{Ca}^{2+}\right]$ (Table 1) as in the previous study [10]. In the presence of $\mathrm{Ca}^{2+}$, stiffness increased later showing $\mathrm{Ca}^{2+}$-dependent contraction. The late contraction at the intermediate $\left[\mathrm{Ca}^{2+}\right]$ was transient as has been reported [8].

The stiffness transients in the presence of $0.63 \mathrm{~mm}$ ADP are illustrated in the lower panel of the same figure (Fig. 1). Two effects of ADP could be seen in the ATP-induced transients of stiffness. Firstly, ADP slowed the rate of decrease in stiffness immediately after the release of ATP. As shown in Table 1, the extent of this effect was independent of $\left[\mathrm{Ca}^{2+}\right]$, and thus the rate of initial stiffness decay was still independent of $\left[\mathrm{Ca}^{2+}\right]$ even in the presence of ADP. Because ADP competes

Table 1. Effect of ADP at various $\left[\mathrm{Ca}^{2+}\right]$ on the rate of the ATP-induced initial decrease in stiffness.*

\begin{tabular}{ccc}
\hline \multirow{2}{*}{$\mathrm{pCa}$} & \multicolumn{2}{c}{$t_{70 \%}{ }^{* *}(\mathrm{~ms})$ at $0.84 \mathrm{mM} \mathrm{ATP}$} \\
\cline { 2 - 3 }$\approx 4.5$ & Control & $+0.63 \mathrm{mM} \mathrm{ADP}$ \\
\hline 6.6 & $15.8( \pm 0.4)$ & $33.5( \pm 1.3)$ \\
$>8\left(+\mathrm{P}_{\mathrm{i}}\right)^{* * *}$ & $14.8( \pm 0.4)$ & $30.2( \pm 0.6)$ \\
\hline
\end{tabular}

* The means ( \pm SEM) of 5 observations are presented. The experiment was the same as that shown in Fig. 1. ** Time for stiffness to decrease to $70 \%$ of the rigor level. *** The solution for the lowest $\left[\mathrm{Ca}^{2+}\right]$ contained $12 \mathrm{mM} \mathbf{P}_{\mathrm{i}}$. 


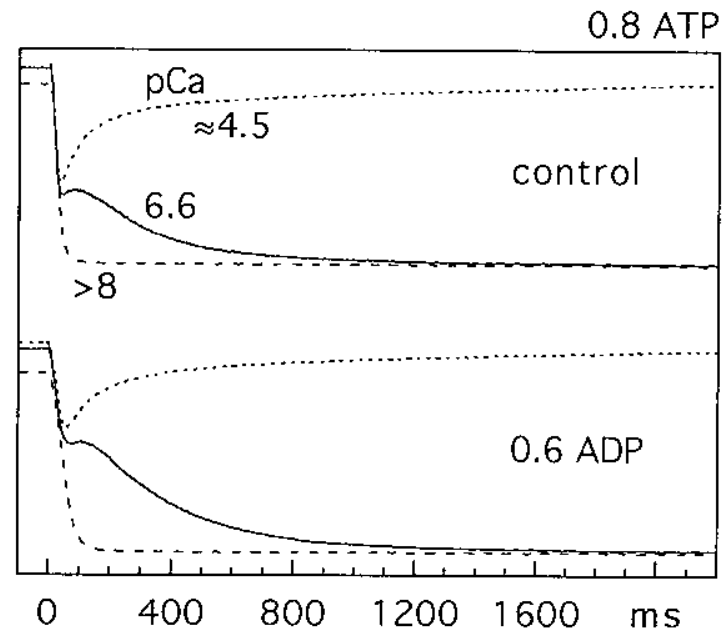

Fig. 1. The ATP-induced transients of stiffness at three different $\left[\mathrm{Ca}^{2+}\right]$, in the absence (upper panel) and presence (lower) of $0.63 \mathrm{mM}$ ADP. ATP (0.84 mM) was photoreleased at time zero. All the photolysis solution contained $10 \mathrm{~mm}$ EGTA, and the amounts of added $\mathrm{CaCl}_{2}$ for $\mathrm{pCa} \approx 4.5,6.6$, and $>8$ were 10 , 3.16 and $0 \mathrm{mM}$, respectively. The $\mathrm{pCa}>8$ solution contained $12 \mathrm{mM} \mathbf{P}_{\mathrm{i}}$. The experiment was made on 5 muscle fibers, changing the order of trials, and the data shown were obtained by averaging.

with ATP in the ATP-induced mechanical transient [1,11], this effect of ADP was probably due to such competition.

In a $\mathrm{Ca}^{2+}$-free ( $10 \mathrm{~mm}$ EGTA) experiment with three muscle fibers, we varied the amount of released ATP and measured the halftime of the monotonic stiffness decrease. On average, the halftime in the absence of ADP was 27, 42, and $79 \mathrm{~ms}$ when $0.96,0.48$, and $0.24 \mathrm{mM}$ ATP was photoreleased, respectively. In the presence of $0.60 \mathrm{~mm}$ ADP, the corresponding values were 53,90, and $182 \mathrm{~ms}$. Thus, in both cases [halftime] ${ }^{-1}(\approx$ rate) was approximately proportional to [ATP], being consistent with the idea of competition between ADP and ATP. (We added $4.7 \mathrm{~mm}$ $\mathbf{P}_{\mathrm{i}}$ in all the $\mathrm{Ca}^{2+}$-free solutions for the same purpose as described above.)

Another effect of ADP was enhancement of the $\mathrm{Ca}^{2+}$-sensitive, late contraction after the initial phase (Fig. 1). This effect was examined in our previous study [8], and was characterized as a sensitization of the late contraction to $\mathrm{Ca}^{2+}$. This effect could be also explained by the competition of ADP with ATP, because the late contraction was also enhanced when the amount of photoreleased ATP was reduced, which will be described later.

\section{Initial contraction in the presence of ADP}

The third effect of ADP was found in the tension transients. This effect was spectacular when $\left[\mathrm{Ca}^{2+}\right]$ was intermediate and the rigor tension before ATP release was set low, and the experiment in Fig. 2 was carried out under these conditions. In 
the control transient (without ADP) at pCa 6.4 (Fig. 2, a), both tension and stiffness decreased immediately after release of $0.86 \mathrm{~mm}$ ATP. In the presence of $0.63 \mathrm{~mm}$ ADP (Fig. 2, $\mathrm{b}_{1}$ and $\mathrm{b}_{2}$ ), the initial decrease in stiffness was slower and the late contraction was larger than the controls like in Fig. 1 (and Table 1).

The remarkable finding in the experiment in Fig. 2 was that, in the presence of ADP (Fig. 2, $b_{1}$ and $b_{2}$ ), tension increased immediately after release of ATP in the phase where stiffness was rapidly decreasing. A slight tension depression separated the initial tension development from the late one.

The initial and late contractions, which were distinguished in the above tension time courses, had different sensitivities to $\mathbf{P}_{\mathrm{i}}$ (orthophosphate) and BDM (2,3butanedione monoxime [12]). As shown in Fig. 2 (c), when we added $10 \mathrm{mM} \mathbf{P}_{\mathrm{i}}$ to the $\mathrm{Ca}+\mathrm{ADP}$ solution, the time course of stiffness lost its late bump and became almost monotonic. In accordance with this, the late tension development was also abolished in the presence of $\mathbf{P}_{i}$. On the other hand, the initial tension development, at least its early part, was not affected by $\mathbf{P}_{i}$. The initial time course of stiffness was also insensitive to $P_{j}$. Suppression of the contraction with $12 \mathrm{mM}$ BDM (4 sets of data from 2 fibers) resulted in a situation (not shown) similar to that with $P_{i}$, although this concentration of BDM seemed to be less effective than $10 \mathrm{mM} P_{i}$, leaving a small but distinct late contraction.

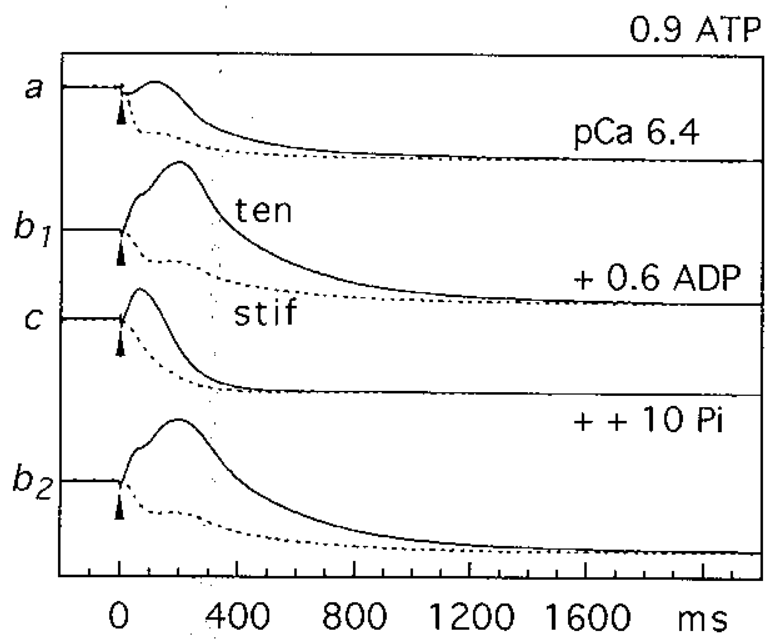

Fig. 2. Paired records of tension (solid line) and stiffness (broken line) on the release of ATP $(0.86 \mathrm{mM})$. All the pairs $\left(\mathrm{a}^{-} \mathrm{c}\right)$ were obtained at pCa $6.4(4.23$ $\mathrm{mm} \mathrm{CaCl}{ }_{2}+10 \mathrm{~mm}$ EGTA). Pairs $b_{1}$ and $b_{2}$ were taken in the presence of 0.63 $\mathrm{mM}$ ADP, and $\mathrm{c}$ in the presence of $10 \mathrm{~mm} \mathrm{P}_{\mathrm{i}}$ with the same amounts of ADP. The peak tensions in $b_{1}$ and $b_{2}$ were about $44 \%$ of the maximum active tension. The data were taken from one fiber in order from top to bottom. The effects of ADP were verified with 5 fibers in total, and the effects of $P_{j}$ with 3 out of the five. 
The difference in $\mathrm{Ca}^{2+}$-sensitivity between the initial and late contractions was obvious in the experiment in Fig. 3, which was performed in the presence of 0.78 mM ADP. At $\mathrm{pCa} \approx 4.5$, the late contraction rose to a plateau after the initial contraction. However, in the absence of $\mathrm{Ca}^{2+}(10 \mathrm{mM}$ EGTA; pCa $>8)$, the late contraction was abolished while the initial contraction was left unaffected. Note that both photolysis trails were carried out in the presence of a low concentration of $\mathbf{P}_{\mathrm{i}}(3.8 \mathrm{mM})$ to make the initial contraction clear. Similar results were obtained with four other fibers.

The magnitudes of the two contractions were affected differently by varying the amount of photoreleased ATP: Reduction in the released ATP suppressed the initial contraction and enhanced the late contraction [8]. All the photolysis trials were performed in the presence of $0.60 \mathrm{~mm}$ ADP. When we reduced the released ATP from 0.96 to $0.24 \mathrm{mM}$, the peak of the late tension at pCa 6.4 with no $P_{i}$ increased by $24 \%$, while the peak of the initial tension at $\mathrm{pCa}>8$ and $4.7 \mathrm{mM} \mathbf{P}_{\mathrm{i}}$ decreased by $20 \%$ (average of three fibers). Similar but smaller effects of $0.48 \mathrm{~mm}$ ATP were also observed in another experiment (two muscle fibers). The peak size of the initial tension was measured as will be described later.

\section{Test of strain-dependence of the mechanical transients}

Dantzig and colleagues [1] suggested that the initial tension development might be caused by possible strain-dependence of the ATP-induced cross-bridge detachment (see DISCUSSION). Thus, we examined whether or not the size of rigor tension before ATP release influenced the mechanical transient immediately

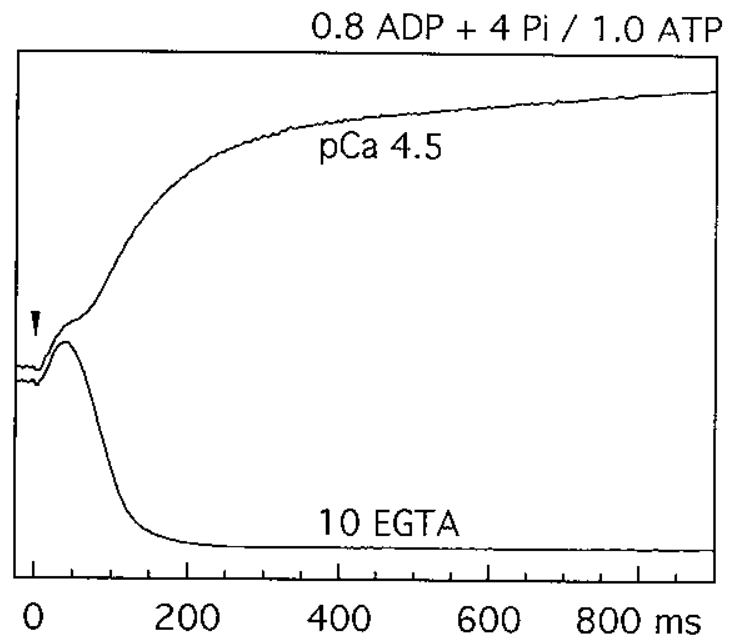

Fig. 3. Minimal effect of $\mathrm{Ca}^{2+}$ on the initial tension development. ATP $1.0 \mathrm{~mm}$ was released at $\mathrm{pCa} \approx 4.5(10 \mathrm{mM}$ Ca.EGTA) and $>8(10 \mathrm{~mm}$ EGTA $)$. In both cases, the photolysis solution contained $0.78 \mathrm{~mm}$ ADP and $3.8 \mathrm{mM} \mathbf{P}_{\mathrm{i}}$. The two tension records were obtained in successive trials with one fiber. 
after the release of ATP. We could detect no great strain-dependence as follows.

The experiment in Fig. 4 was made on 5 muscle fibers releasing $0.65 \mathrm{mM}$ ATP in the presence of $1.43 \mathrm{mM}$ ADP and $7.0 \mathrm{mM} P_{i}$ in the absence of $\mathrm{Ca}^{2+}(10 \mathrm{~mm}$ EGTA). The high concentration of ADP was to saturate the cross-bridges with ADP. To change the cross-bridge strain, the muscle in rigor was stretched before release of ATP in one trial and kept isometric in the other trial. The isometric rigor tension was about $35 \%$, on average, of the maximum active tension, and stretching increased this to $92 \%$ of the maximum. There was a shoulder or a bump on both tension time courses, indicating the initial contraction in the presence of ADP.

The time courses of stiffness change in the two trials (Fig. 4, stif) were almost identical, with no sign of strain-dependence of the rate of the initial reaction. It should be noted that the rigor stiffness was $\approx 30 \%$ higher in the trial with stretch than in the isometric one, probably because of the presence of the non-linear end-compliance (see DISCUSSION). The halftime for the stiffness decrease was $113( \pm 7) \mathrm{ms}(n=5)$ in the isometric trial, and this was only $4( \pm 5) \%$ larger than

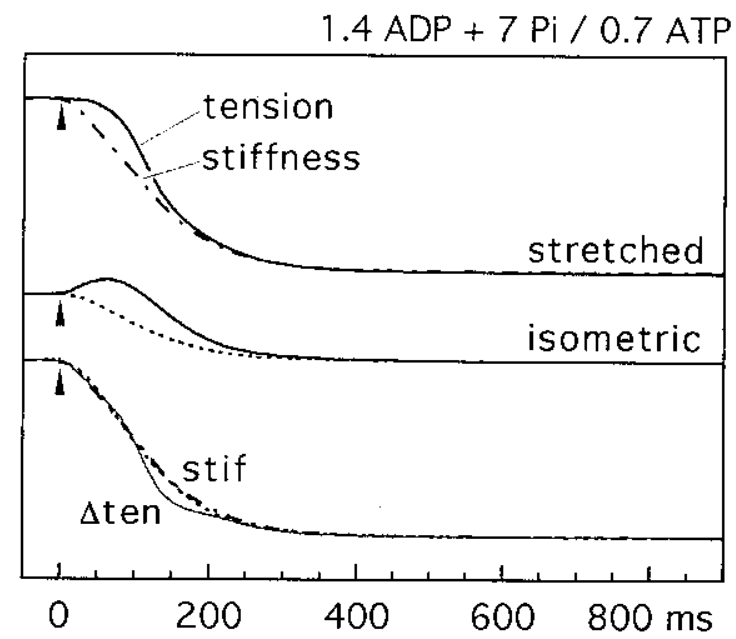

Fig. 4. Time courses of tension (solid line) and stiffness (broken line) on release of $0.65 \mathrm{mM}$ ATP in the absence of $\mathrm{Ca}^{2+}$ (10 mM EGTA). ADP $1.43 \mathrm{mM}$ and 7.0 mM $P_{i}$ were added in the caged ATP solution. In one trial (top) the muscle fiber was stretched by about $1 \% 2 \mathrm{~s}$ before ATP release, while in the other trial (middle) the fiber was kept isometric. In the upper two panels, each stiffness record was scaled so that before release of ATP its height is the same as that of the corresponding tension record. In the bottom panel, the two stiffness records were scaled so as to have the same amplitude (stif). The original rigor stiffness with stretch was $28 \%$ larger than the isometric one. The rigor tension with stretch was $92 \%$ of the maximum active tension. The data were obtained by averaging 5 sets of records from different fibers. See the legend of Fig. 7 for $\Delta$ ten. 
in the trial with stretch. Note that the halftime was as short as $39( \pm 1.2) \mathrm{ms}$ in the absence of ADP (not shown in Fig. 4), regardless of whether the fiber was stretched or kept isometric.

We evaluated the magnitude of the developed tension assuming that the stiffness decrease represented the time course of degradation of the rigor tension with ATP binding. We superimposed the stiffness trace on the tension record scaling the former as in Fig. 4 (top and middle), and measured the height of tension above the stiffness trace. The peak of the tension above stiffness in the stretch trial was $96( \pm 3) \%(n=5)$ of that in the isometric trial.

\section{$A D P$-dependence of the initial mechanical transient}

The halftime for the stiffness decrease and the magnitude of the initial contraction were dependent on [ADP], as shown in Fig. 5. The amount of released ATP was $0.76 \mathrm{~mm}$. The halftime (Fig. 5A) increased monotonically with increasing [ADP], and at $1.81 \mathrm{~mm}$ ADP this was about 3 times longer than the control. The presence of $8.9 \mathrm{~mm} P_{i}$ in the photolysis solutions slightly shortened the stiffness halftime (by about 10\%; Fig. 5A, $O$ vs. - ), which could be due to suppression of the ADP-enhanced late contraction. We analyzed these data based on the following simplified Scheme 2 , taking account of the finite rate $\left(\approx 40 \mathrm{~s}^{-1}\right)$ of ATP release in photolysis [5]:

$$
\mathrm{ADP} \cdot \mathrm{R} \underset{k_{+\mathrm{D}} \cdot \mathrm{ADP}}{\stackrel{k_{-\mathrm{D}}}{\rightleftarrows}} \mathrm{R} \stackrel{k_{+\tau} \cdot \mathrm{ATP}}{\longrightarrow} \mathrm{W}
$$

The rate constants estimated from the analysis were as follows: $k_{+\mathrm{T}}=5.3 \times 10^{4} \mathrm{M}^{-1}$ $\mathrm{s}^{-1}, k_{-\mathrm{D}}=29 \mathrm{~s}^{-1}, k_{+\mathrm{D}}=5.7 \times 10^{4} \mathrm{M}^{-1} \mathrm{~s}^{-1}$, and thus $K_{\mathrm{D}}\left(=k_{-\mathrm{D}} / k_{+\mathrm{D}}\right)=0.51 \mathrm{mM}$. According to Sleep and colleagues [13], caged ATP binds to the cross-bridges with $K_{\mathrm{i}} \approx 1.6 \mathrm{mM}$. We released $0.76 \mathrm{mM}$ ATP from $4.5 \mathrm{mM}$ caged ATP in these particular experiments, so that in the above $\left(k_{+\mathrm{T}}\right.$ and $\left.k_{+\mathrm{D}}\right)$ and $K_{\mathrm{D}}$ would be under- and overestimated, respectively, by a factor of $\approx 3.3(=1+(4.5-0.76) / 1.6)$.

The peak size of the initial contraction increased with the increase in [ADP] as in Fig. 5B, and the shape of the concentration-response curve was not greatly affected by $8.9 \mathrm{~mm} \mathrm{P}_{\mathrm{i}}(\bigcirc \square$ vs. $+\bullet$ ). The peak size at $1.81 \mathrm{~mm}$ ADP (with $0.76 \mathrm{~mm}$ ATP) was $15( \pm 1.5) \%(n=12)$ of the maximum active tension. The fitting of a simple hyperbolic curve to the data resulted in the apparent $K_{\mathrm{m}}$ of $0.08 \mathrm{mM}$ for ADP.

\section{Possible effect of contaminant ATP in the solutions}

One may suspect that the initial contraction in the presence of ADP might be somehow due to contamination of ATP in the ADP solution. The cross-bridge reaction may be cycling very slowly with the small amount of ATP even before photorelease of ATP. A significant amount of cross-bridges might be in state F (Scheme 1) dragging other cross-bridges in state ADP $\cdot R$. On photorelease of ATP, the passive force of the ADP $R$ cross-bridges disappears, so that the active 


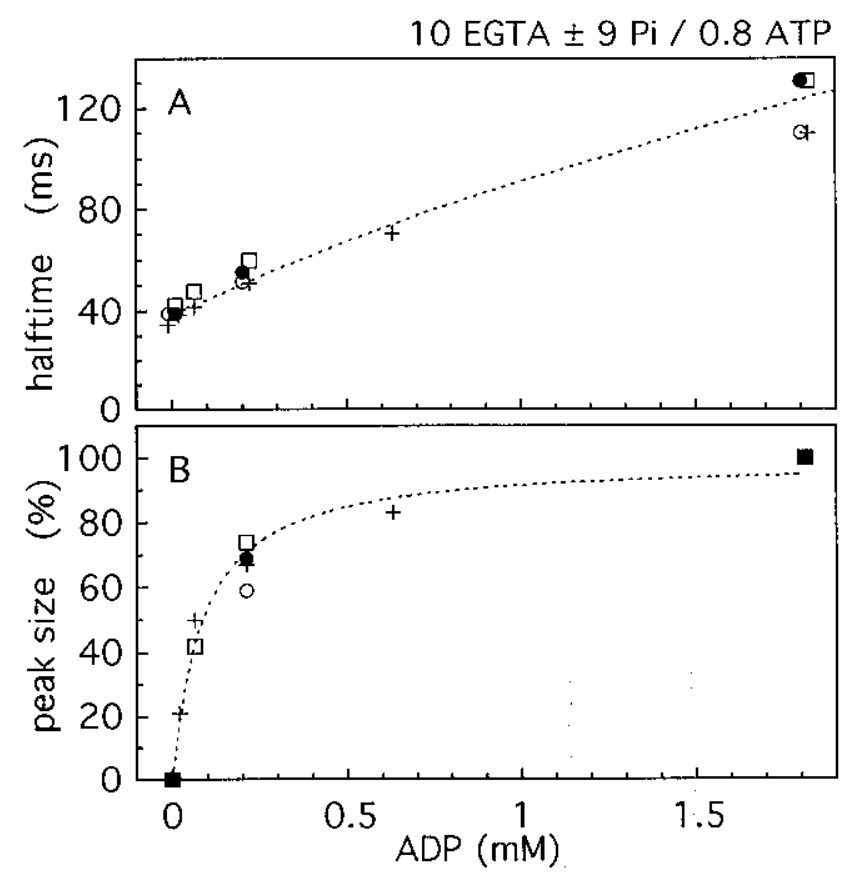

Fig. 5. The halftime of stiffness decay (A) and the magnitude of initial contraction (B) as functions of [ADP]. ATP $0.76 \mathrm{mM}$ was released from $4.5 \mathrm{mM}$ of caged ATP in the absence of $\mathrm{Ca}^{2+}$ (10 mM EGTA). The different symbols represent different experiments, each being with 5-6 muscle fibers. Two experiments $(+$, -) were carried out without $P_{i}$ and two $(O, \square)$ in the presence of $8.9 \mathrm{~mm} \mathrm{P}_{\mathrm{i}}$. The muscle fibers in the experiments with - and $O$ were the same. In $A$, the broken line represents the data calculated in the kinetic model described in the text (Scheme 2). In B, the peak size was normalized so that it is $100 \%$ at 1.81 $\mathrm{mM}$ ADP and $0 \%$ without ADP. The broken line is peak $=$ peak $_{\max } /\left(1+K_{\mathrm{m}} /\right.$ ADP), fitted to the data.

force of the F cross-bridges would be uncovered. In the experiment shown in Fig. 6, we added a small amount of ATP to the ADP solution to examine such possibilities.

The experiment was carried out under the $\mathrm{Ca}^{2+}$-free conditions in the presence of $\mathbf{P}_{j}$. In Fig. 6, the obtained tension transients on release of ATP are superimposed with appropriately scaled stiffness traces to inspect the sizes of initial contractions. In the control without ADP (Fig. 6, a), tension and stiffness decreased with almost the same time course, showing little sign of the initial contraction. When we added $60 \mu \mathrm{M}$ ADP in the caged ATP solution (Fig. 6, b), the initial contraction appeared. This initial contraction did not seem to be due to conversion of two ADPs into ATP plus AMP by the myokinase intrinsic in the muscle fibers, because the inhibitor of the kinase, $\mathrm{AP}_{5} \mathrm{~A}$ [14], had no effect on the result even at $0.1 \mathrm{mM}$ (Fig. 6, c). 


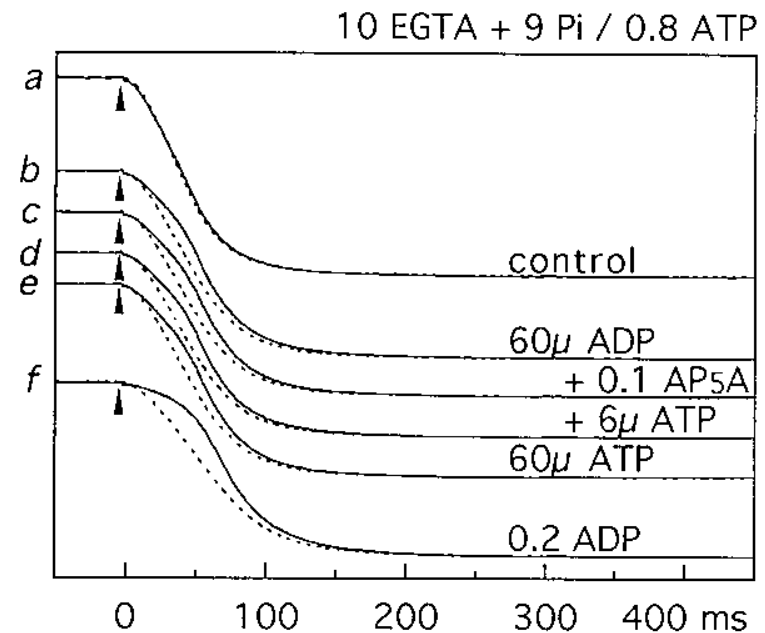

Fig. 6. Minimal effect of contaminant ATP on the initial contraction. All the photolysis solutions contained $4.5 \mathrm{mM}$ caged ATP, $8.9 \mathrm{mM} \mathrm{P}_{\mathrm{i}}$, and $10 \mathrm{mM}$ EGTA with no added $\mathrm{Ca}^{2+}$, and $0.75 \mathrm{~mm}$ ATP was released at time zero. The solid lines are the tension records. The broken lines are the stiffness traces which were scaled so that their pre-photolysis levels are the same as the corresponding tension records. The caged ATP solution contained no added nucleotides in a, $60 \mu \mathrm{M}$ ADP in b, $60 \mu \mathrm{M}$ ADP plus $0.1 \mathrm{mM} \mathrm{AP} \mathrm{P}_{5} \mathrm{~A}$ in c, $60 \mu \mathrm{M}$ ADP plus $6 \mu \mathrm{M}$ $\mathrm{ATP}$ in $\mathrm{d}, 60 \mu \mathrm{M}$ ATP in e, and $0.2 \mu \mathrm{M} \mathrm{ADP}$ in $\mathrm{f}$. The incubation time in the caged ATP solutions was $30 \mathrm{~s}$ in all the cases. In c, $\mathrm{AP}_{5} \mathrm{~A}$ was introduced $30 \mathrm{~s}$ before introduction of ADP. Each pair of records was obtained by averaging 5 pairs from 5 fibers. The muscle fibers were stretched by $1 \%, 5 \mathrm{~s}$ before ATP release.

The addition of $6 \mu \mathrm{M}$ ATP to the ADP solution (Fig. 6, d) did not remarkably enhance the initial contraction. Probably the cross-bridge ATPase in the fiber hydrolyzed ATP into ADP during the incubation before photolysis. When we replaced the $60 \mu \mathrm{M}$ ADP with the same amount of ATP (Fig. 6, e), the fiber developed a significant force during the incubation time, but the size of the initial contraction was almost the same as the control with $60 \mu \mathrm{M}$ ADP. Therefore, the small amount of ATP contaminating our ADP (ATP/ADP $<0.2 \%$ ) did not seem to be the cause of the initial contraction. It should be noted that the size of the initial contraction was several times larger at $0.2 \mathrm{mM}$ ADP (Fig. 6, f) than at $60 \mu \mathrm{M}$ as has been shown in Fig. 5.

\section{Time course of "tension difference"}

In the previous studies $[1,11]$ the method of tension difference was employed to estimate the time course of cross-bridge "detachment" (ADP $R+A T P \rightarrow W+$ $\mathrm{ADP}$ ) on release of ATP. In the stretching experiments, such as shown in Figs. 4 
and 7 , the two records with the different rigor tensions approached each other in time after ATP release. It is likely that cross-bridges lose the initial rigor force on detachment. Thus, it is reasonable to assume that the time course of the tension difference ( $\Delta$ ten), i.e. the algebraic difference between the two records, represented the time course of the rigor degradation. However, it turned out that $\Delta$ ten was not always reliable:

The time courses of stiffness, tension and $\Delta$ ten were compared in the experiment in Fig. 7. $\left[\mathrm{Ca}^{2+}\right]$ was set at an intermediate concentration (pCa 6.4) so as to attenuate the late contraction. Because of the presence of $0.63 \mathrm{~mm} \mathrm{ADP}$, the initial contraction preceded the late contraction. The time course of stiffness change (Fig. 7 , lower) was not greatly affected by the rigor tension before ATP release, like in the absence of $\mathrm{Ca}^{2+}$ (Fig. 4), and consisted of three phases: (i) the fast decrease during the initial contraction, (ii) the following upward deflection due to the late contraction, and (iii) the final decay to the baseline due to the transient nature of the contraction.

The time course of $\Delta$ ten (Fig. 7) was smoother than that of stiffness, but not ideally monotonic. Its initial time course was roughly the same as that of stiffness (i), which is consistent with the theoretical idea. However, the later time course of $\Delta$ ten had a significant bump which temporally coincided with the late phases (ii-iii)

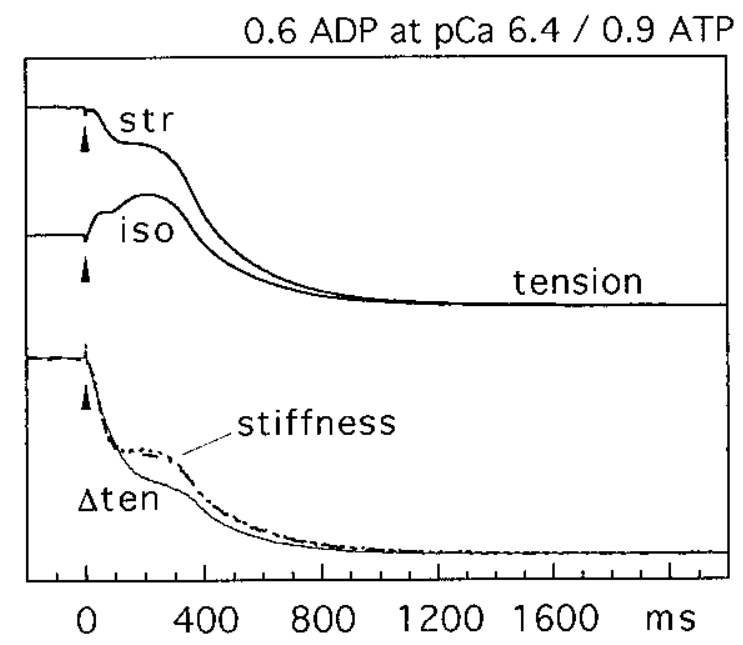

Fig. 7. Tension (solid line) and stiffness (broken line) on release of $0.86 \mathrm{~mm}$ ATP at $\mathrm{pCa} 6.4$ in the presence of $0.63 \mathrm{~mm}$ ADP. The fiber was stretched by $1 \%$ before ATP release in one trial (str), and kept isometric in the other trial (iso). The tension difference ( $\Delta$ ten $=s t r-i s o$ ) is superimposed on the stiffness records. Note that stiffness and $\Delta$ ten traces are scaled so as to have the same amplitudes. The rigor tension in the trial with stretching was $90 \%$ of the maximum active tension. The rigor stiffness in the stretching trial was $33 \%$ higher than that in the isometric trial. 
in the stiffness records. This deviation of $\Delta$ ten from the ideal time course was confirmed with other three fibers. These time courses of $\Delta$ ten at the intermediate $\left[\mathrm{Ca}^{2+}\right]$ were similar to those obtained by Dantzig and colleagues [1] in the absence of $\mathrm{Ca}^{2+}$.

The shape of the $\Delta$ ten time course under the $\mathrm{Ca}^{2+}$-free conditions (Fig. 4) was different from the above. Its later half was convex downward (Fig. 4), and showed large fiber-to-fiber variation (not shown). We also examined $\Delta$ ten's at the supramaximal $\left[\mathrm{Ca}^{2+}\right](\mathrm{pCa} \approx 4.5)$ with two fibers, and confirmed that they were relatively smooth as in the previous studies [1,11].

As mentioned earlier, the initial phase of the ATP-induced reaction was not significantly affected by $\mathrm{Ca}^{2+}$, judging from the time courses of tension and stiffness. The initial time course of $\Delta$ ten was also insensitive to $\mathrm{Ca}^{2+}$. The experiment was performed with two muscle fibers at three different $\left[\mathrm{Ca}^{2+}\right]: \mathrm{pCa}>8,6.4$, and $\approx 4.5$. The amount of released ATP was relatively small $(0.41 \mathrm{~mm})$ so as to slow the binding of ATP. The halftime of $\Delta$ ten in the absence of ADP was $50-60 \mathrm{~ms}$ irrespective of $\left[\mathrm{Ca}^{2+}\right]$, and similar to the stiffness halftime $(68 \pm 3 \mathrm{~ms}$; mean $\pm \mathrm{SD}$, 6 trials). The $\Delta$ ten halftime in the presence of $0.63 \mathrm{~mm}$ ADP were about 215,200 , and $185-200 \mathrm{~ms}$ at $\mathrm{pCa}>8,6.4$, and $\approx 4.5$, respectively, and again similar to the stiffness halftime at pCa $>8,200-205 \mathrm{~ms}$. Thus, we saw no substantial $\mathrm{Ca}^{2+}$-dependence of the cross-bridge "detachment."

\section{DISCUSSION}

In the present and previous [8] studies, we examined the effects of ADP on the mechanical transient initiated by photorelease of ATP in rigor muscle fibers. There were three effects of ADP on the transient: 1) ADP slowed the initial decrease in stiffness immediately after the release of ATP. This was probably from competition between ADP and ATP [1,11] in the reaction of nucleotide replacement (ADP. $\mathbf{R} \stackrel{-A D P}{\longrightarrow} \mathbf{R} \stackrel{+A T P}{\longrightarrow} W)$. The rate of this reaction was not greatly affected by $P_{i}, B D M$, $\mathrm{Ca}^{2+}$, or the pre-photolysis rigor tension. 2) ADP enhanced the late, $\mathrm{Ca}^{2+}$-sensitive development of tension and stiffness that has been considered [15] to occur with the release of $\mathbf{P}_{\mathrm{i}}$ after ATP hydrolysis $\left(\mathrm{W} \rightarrow \mathrm{F}+\mathbf{P}_{\mathrm{i}}\right)$. This effect of ADP was discussed in our previous study [8]. 3) Only in the presence of ADP did the muscle fiber develop tension during the initial phase of the ATP-induced reaction (ADP. $\mathbf{R} \stackrel{-A D P}{\longrightarrow} \mathbf{R} \stackrel{+A T P}{\longrightarrow} \mathbf{W})$. Unlike the late contraction with $\mathbf{P}_{i}$ release, this "initial contraction" was not sensitive to $\mathrm{Ca}^{2+}, \mathrm{P}_{\mathrm{i}}$, or $\mathrm{BDM}$. It was also independent of the pre-photolysis rigor tension.

Cross-bridge "detachment" induced by $A T P$. It has been implied $[1,5,6]$ that the cross-bridges are substantially detached in the weak-binding state (W) in the initial reaction after the binding of ATP (Scheme 1). This "detachment" monitored with stiffness in the absence of $\mathrm{Ca}^{2+}$ was slowed by ADP. Although the tension difference ( $\Delta$ ten) was not always reliable, it also suggested the same ADP effect that stiffness indicated. 
The ADP-dependence of the halftime of the detachment-like reaction (Fig. 5A) was simpler than that obtained by Dantzig and colleagues (Fig. 6A of Dantzig et al. [1]). We carried out the experiments at $8^{\circ} \mathrm{C}$ and took the stiffness records for analysis of the effect of ADP. In the absence of $\mathrm{Ca}^{2+}$, stiffness showed monotonic time courses under our conditions, so that we measured the halftime of the overall time course. On the other hand, the previous authors [1] performed experiments at $21^{\circ} \mathrm{C}$ and took $\Delta$ ten for analysis. The time courses of $\Delta$ ten under their conditions were similar to or more complex than ours (Figs. 4 and 7), and they measured the halftime of the late phase of the complex time course.

In this study, the initial time course of stiffness indicated that $\mathrm{Ca}^{2+}$ does not affect the detachment-like reaction even in the presence, as well as the absence [10], of ADP (Fig. 1 and Table 1). $\mathrm{Ca}^{2+}$ seemed to be a simple inducer of the late contraction. Dantzig and colleagues [1], from their analysis of $\Delta$ ten, suggested that $\mathrm{Ca}^{2+}$ doubled the rate of "etachment" in the presence of ADP (Fig. 6A of Dantzig et al. [1]). In this study, the initial time course of $\Delta$ ten was independent of $\left[\mathrm{Ca}^{2+}\right]$, like that of stiffness, even in the presence of $0.6 \mathrm{mM}$ ADP.

Strain-dependence of "detachment". Dantzig and colleagues [1] inferred the strain-dependent detachment of ADP-rigor cross-bridges from the result of their "negative tension" experiment (Fig. 11 of Dantzig et al. [1]), which was similar to the one in Fig. 4 but was carried out at a long sarcomere length. Because of the high resting tension in this situation, they could set the rigor tension lower than the resting level, by releasing rigor fibers. When ATP was photoreleased in the presence of ADP, tension rapidly rose to the resting level in the negative-tension trials, while it slowly fell to this level in the normal, isometric trials. The difference between the two rates was interpreted to mean that the ADP $\mathbf{R}$ cross-bridges reached the detached state (W) faster when the cross-bridge strain was lower or more negative.

Danzig and colleagues added $\mathbf{P}_{\mathrm{i}}$ or $\mathrm{BDM}$ in the photolysis solutions to inhibit the contraction $\left(\mathrm{W} \rightarrow \mathrm{F}+\mathbf{P}_{\mathrm{i}}\right)$. However, the initial contraction probably remained in their transient. Thus, the "relaxation" in their negative-tension trials could be the rising phase of the initial contraction in this paper, while that in the isometric trials could be its falling phase. Therefore, the result of their negative tension experiment does not seem to evidence the strain-dependent behavior of the ADP. $\mathbf{R}$ cross-bridges.

To test the strain-dependence, we compared the time courses of stiffness in the two photolysis trials: the fiber was kept isometric in one trial and stretched in the other (Figs. 4 and 7). The two time courses were almost identical to each other. Because of the presence of the non-linear end-compliance, which is more compliant at lower tension, it is likely that the muscle stiffness at lower tension was underestimated in our experiments [6]. Therefore, the identical time courses of our stiffness in the two trials above implies that the actual muscle stiffness decreased faster when the muscle was more strained. This strain-dependence is in the direction opposite to what was suggested in the negative tension experiment. The

Vol. 44, No. 6, 1994 
opposite strain-dependence has been observed in other studies of $\Delta$ ten $[5,11]$.

Initial contraction during "detachment". In the presence of ADP, the initial reaction on release of ATP was a contraction where tension per stiffness $(500 \mathrm{~Hz})$ appeared to be relatively high. When we released $0.8 \mathrm{mM}$ ATP in the presence of $1.8 \mathrm{mM} \mathrm{ADP}$, the size of the tension which developed during the initial contraction was about $15 \%$ of the maximum steady tension. The size became small as the amount of photoreleased ATP was reduced. Probably, the magnitude of the initial contraction is larger when the rate at which the ADPs on the cross-bridges are replaced with ATPs is faster. This rate-dependence may be the reason why $K_{\mathrm{m}}$ for ADP in Fig. 5B $(0.08 \mathrm{~mm})$ was lower than $K_{\mathrm{D}}$ in Fig. $5 \mathrm{~A}(0.5 \mathrm{mM})$ : When we increased [ADP] keeping [ATP] constant, the ADP-ATP replacement was slowed and this, in turn, reduced the size of the initial contraction.

Dantzig and colleagues [1] found in their $\mathrm{Ca}^{2+}$-experiments (Fig. 17 of Dantzig et al. [1]) that the initial tension decrease in the presence of ADP was much smaller than expected from the simple model (Scheme 1). Judging from our result in Fig. 3, the previous authors' observation seems to be closely related with the initial contraction we examined in this study. They proposed several possible mechanisms to explain their results. In the following, we consider if the proposed mechanisms or others can explain our results without assuming that the nucleotide replacement induces actual force generation before the release of $\mathbf{P}_{\mathrm{i}}$.

(1) The mechanism that the previous authors [1] mentioned first was the strain-dependent ADP-release from the ADP $\cdot R$ cross-bridges (ADP $\cdot R \rightarrow A D P+$ $R$ ). The ADP $\cdot R$ cross-bridges might release ADP faster when they are negatively strained than when they are positively strained. If so, in the early phase of the ATP-induced detachment, the breakdown of the negatively strained cross-bridges results in an increase in the fiber tension.

There is, however, no clear evidence for the fast detachment of the negatively strained rigor cross-bridges, as discussed above. Furthermore, we could not detect such a strain-dependent detachment, but could observe the initial contraction that is not strain-dependent. Because the low rigor tension in our experiment was not negative, it was still possible that the amount of the negatively strained crossbridges was too small to be detected in the time course of stiffness. Even if this was the case, the initial contraction detected in the tension time course would have been strain-dependent under the hypothesis. An analysis of our results with a quantitative model in APPENDIX also failed to support the strain-dependence mechanism.

(2a) Another mechanism suggested previously [1] was the cooperative interaction between the cross-bridges. When one of the two myosin heads is of ADP. $R$ and the other one $W$, the force-generating transition of the $W$ head $\left(W \rightarrow F+P_{i}\right)$ might be accelerated by the ADP $R$ partner. However, this cooperation does not seem compatible with our observation that the initial contraction is resistant to $\mathbf{P}_{\mathbf{i}}$ and BDM. (2b) Another type of cooperation was also considered previously [1]. The pairs of $\mathrm{W}$ and ADP $\cdot \mathrm{R}$ cross-bridges might keep the same tension they had 
before detachment of the $\mathrm{W}$ head. This mechanism explains the maintenance of tension but not the increase in tension on detachment.

(3) The addition of ADP to the rigor fiber reduces its tension [1]. The apparent $K_{\mathrm{m}}$ of $\mathrm{ADP}$ for this effect was about $0.1 \mathrm{mM}$ under our experimental conditions (Horiuti, unpublished observation) and $0.02 \mathrm{mM}$ in the study by Dantzig and colleagues [1]. However, its simple reversal (ADP $R \stackrel{R}{\longrightarrow} \mathrm{ADP}$ ) in the ATPinitiated reaction (ADP $\cdot R \stackrel{-A D P}{\longrightarrow} R \stackrel{+A T P}{\longrightarrow} W$ ) would not be the cause of the initial contraction. Firstly, the decrease in tension by ADP is only $10-20 \%$ of the rigor tension ([1]; Horiuti, unpublished observation). Secondly, the initial contraction became larger when the $\mathrm{R}$ cross-bridges disappeared faster in the presence of larger amounts of ATP (this study).

In the above, we considered several possible mechanisms (1-3) for the initial contraction, but none of them were satisfactory. Instead, our experimental results with the $\mathrm{Ca}^{2+}$ - and $\mathrm{P}_{\mathrm{i}}$-insensitive, rapid tension development on release of ATP seem to suggest that the individual cross-bridges generate contractile force during or immediately after the replacement of ADP with ATP before the other process of force generation starts with the release of $P_{i}$. Both processes of force generation should be investigated to understand the contractile mechanism of the cross-bridge reaction.

This study was supported by a Grant-in-Aid for Scientific Research from the Ministry of Education, Science and Culture, Japan, and grants from Ciba-Geigy Foundation (Japan) for Promotion of Sciences, Terumo Life Science Foundation, and Ichiro Kanehara Foundation.

\section{REFERENCES}

1. Dantzig JA, Hibberd MG, Trentham DR, and Goldman YE: Cross-bridge kinetics in the presence of MgADP investigated by photolysis of caged ATP in rabbit psoas muscle fibres. J Physiol (Lond) 432: 639-680, 1991

2. Dantzig JA, Goldman YE, Millar NC, Lacktis J, and Homsher E: Reversal of the cross-bridge force-generating transition by photogeneration of phosphate in rabbit psoas muscle fibres. J Physiol (Lond) 451: 247-278, 1992

3. Kawai $M$ and Halvorson HR: Two step mechanism of phosphate release and the mechanism of force generation in chemically skinned fibers of rabbit psoas muscle. Biophys J 59: 329-342, 1991

4. Millar NC and Homsher E: The effect of phosphate and calcium on force generation in glycerinated rabbit skeletal muscle fibers. J Biol Chem 265: 20234-20240, 1990

5. Goldman YE, Hibberd MG, and Trentham DR: Relaxation of rabbit psoas muscle fibres from rigor by photochemical generation of adenosine-5'-triphosphate. J Physiol (Lond) 354: 577-604, 1984

6. Goldman YE, Hibberd MG, and Trentham DR: Initiation of active contraction by photogeneration of adenosine-5'-triphosphate in rabbit psoas muscle fibres. J Physiol (Lond) 354: 605-624, 1984

Vol. 44, No. 6, 1994 
7. Horiuti $\mathrm{K}$, Kagawa $\mathrm{K}$, and Yamada $\mathrm{K}$ : Three effects of ADP on the ATP-induced transient in skinned muscle fibers. Jpn J Physiol 44: S143, 1994 (abstract)

8. Horiuti $\mathrm{K}$, Kagawa $\mathrm{K}$, and Yamada $\mathrm{K}$ : Transient contraction of muscle fibers on photorelease of ATP at intermediate concentration of $\mathrm{Ca}^{2+}$. Biophys J 67: 1925-1932, 1994

9. Kawai $\mathbf{M}$ and Brandt PW: Sinusoidal analysis: A high resolution method for correlating biochemical reactions with physiological processes in activated skeletal muscles of rabbit, frog and crayfish. J Muscle Res Cell Motil 1: 279-303, 1980

10. Horiuti K, Sakoda T, and Yamada K: Time course of rise of muscle stiffness at onset of contraction induced by photorelease of ATP. J Muscle Res Cell Motil 13: 685-691, 1992

11. Horiuti K, Sakoda T, and Yamada K: Mechanical response to photolytic ATP pulses of skinned muscle fibres pre-activated with a small pulse of ATP. J Muscle Res Cell Motil 14: 335-340, 1993

12. Horiuti K, Higuchi $\mathrm{H}$, Umazume $\mathrm{Y}$, Konishi M, Okazaki $\mathrm{O}$, and Kurihara S: Mechanism of action 2,3-butanedione 2-monoxime on contraction of frog skeletal muscle fibres. J Muscle Res Cell Motil 9: 156-164, 1988

13. Sleep J, Herrman C, Barman T, and Travers F: Inhibition of ATP binding to myofibrils and acto-myosin subfragment 1 by caged ATP. Biochemistry 33: 6038-6042, 1994

14. Lienhard GE and Secemski II: $\mathbf{P}^{1}, \mathrm{P}^{5}-\mathrm{Di}\left(\right.$ adenosine- $\left.5^{\prime}\right)$ pentaphosphate, a potent multisubstrate inhibitor of adenylate kinase. J Biol Chem 248: 1121-1123, 1973

15. Hibberd MG, Dantzig JA, Trentham DR, and Goldman YE: Phosphate release and force generation in skeletal muscle fibers. Science 228: 1317-1319, 1985

\section{APPENDIX}

In this section, we assume that the initial contractions in the experiment in Fig. 4 were caused by the mechanism that the negatively strained rigor cross-bridges detach much more rapidly than the positively strained ones. Based on this assumption, we estimate the amount of the negatively strained cross-bridges in the isometric trial and how much of this should have remained in the stretch trial in order to determine the expected change in the size of the initial contraction.

We take a Gaussian for the population density of the cross-bridges as regards the strain, and assume that the stress is proportional to the strain. The mean stress measured by the standard deviation is denoted by $\mu$. If the value of $\mu$ is given, we can calculate the fraction $\left(f^{-}\right)$of the negatively strained cross-bridges and the mean of the negative stress $\left(\mu^{-}\right)$, as shown in Fig. 8. In the isometric trial in Fig. 4, the size of the initial contraction was about one-half of the rigor tension. Thus, under the strain-dependence hypothesis, $\mu^{-} \approx 0.5 \cdot \mu$, so that $\mu \approx 0.44, \mu^{-} \approx 0.22$, and $f^{-} \approx$ 0.33 (Fig. 8, $\alpha$ and a). In other words, in the isometric trial, one-third of the total cross-bridges seemed to be negatively strained and they must have detached faster than the other, positively strained ones. Why was the decay of stiffness monotonic?

In the stretching trial (Fig. 4), the rigor tension was 2.6 times higher than in the isometric trial. Thus, in the model calculation (Fig. $8, \beta$ and b), $\mu$ is increased 


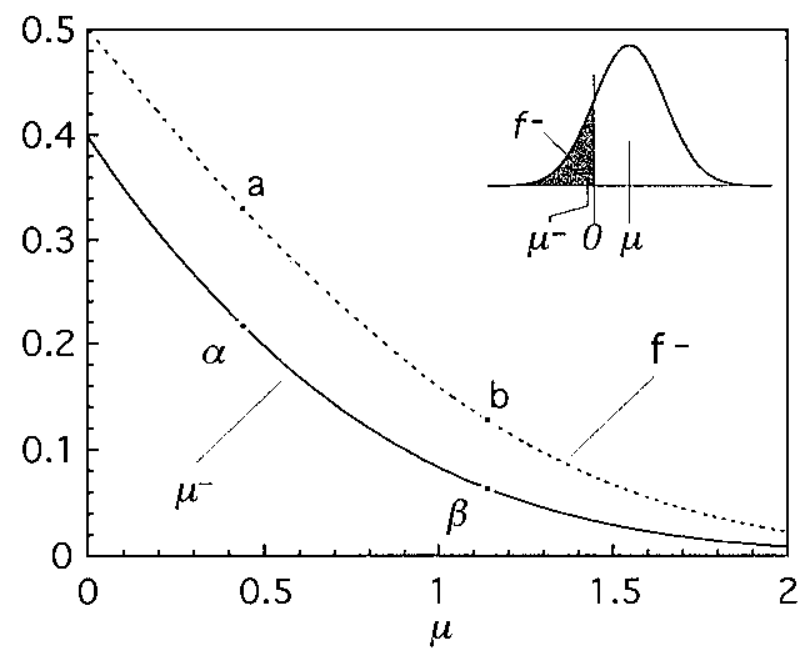

Fig 8. The fiaction $\left(f^{-}\right)$of the cross-bridges negatively strained, and the mean negative stress $\left(\mu^{-}\right)$, both as functions of the mean stres $(\mu)$. The data were calculated as follows: $f^{-}=\int_{\mu}^{\infty} g(x) \mathrm{d} x$ and $\mu^{-}=\int_{\mu}^{\infty}(x-\mu) \cdot g(x) \mathrm{d} x$, where $g(x)=$ $\exp \left(-x^{2} / 2\right) / \sqrt{2 \pi}$

to $1.16(=0.44 \times 2.6)$, which results in a decrease in $f^{-}$to 0.13 , where $\mu^{-}$is only 0.06 . Therefore, the size of the tension developed by the negatively strained cross-bridges could have been reduced to one-quarter $(=0.06 / 0.22)$ by stretching. Why did this not happen in our experiment?

The results of the calculations did not seem to be greatly dependent on the type of the distribution function of the cross-bridge strain. If we take a uniform distribution $(g(x)=0.287,|x|<1.745)$, instead of the Gaussian; $\mu \approx 0.47, \mu^{-} \approx 0.23$, and $f^{-} \approx 0.37$ in the isometric trial; and $\mu \approx 1.22(=0.47 \times 2.6), \mu^{-} \approx 0.04$, and $f^{-} \approx$ 0.15 in the stretch trial. 